\title{
Chronic Partial Ureteral Obstruction in the Neonatal Guinea Pig. I. Influence of Uninephrectomy on Growth and Hemodynamics
}

\author{
ROBERT L. CHEVALIER AND DONALD L. KAISER \\ with the technical assistance of Anthony V. Broccoli \\ Departments of Pediatrics and Internal Medicine, University of Virginia School of Medicine, \\ Charlottesville, Virginia 22908
}

\begin{abstract}
Although obstructive nephropathy is a frequent cause of renal insufficiency in infancy, factors influencing adaptation to chronic partial ureteral obstruction (CPUO) are poorly understood. Guinea pigs were subjected to unilateral CPUO within the first 2 days of life (Group I) and microsphere studies were performed at $23 \pm 3$ days of age. To also investigate the role of functional renal mass on growth and hemodynamics, contralateral nephrectomy was performed in Group II at the time of ureteral constriction. Compared to sham-operated controls (ureteral diameter $=1 \mathrm{~mm}$ ) CPUO caused impaired somatic growth in both groups. Resulting hydroureteronephrosis was variable and most severe (ureteral diameter $>3 \mathrm{~mm}$ ) in azotemic animals of Group II, which demonstrated reduced compensatory renal hypertrophy and poorest somatic growth.

Mean arterial blood pressure was not affected by CPUO or uninephrectomy. Renal blood flow (RBF) was higher in Group II than Group I, and did not fall significantly with severity of CPUO in Group II, in which number of perfused glomeruli was similar to controls. In contrast, renal vascular resistance (RVR) increased by $172 \%$ in animals of Group I with severe CPUO, and was associated with $34 \%$ fewer perfused glomeruli than in the hypertrophied contralateral kidney. With increasing severity of CPUO, RBF tended to be distributed to outer cortical regions in the obstructed kidney of Group I, while the shift in RBF distribution was from outer to inner cortex in the remaining kidney of Group II. Filtration fraction fell as a result of CPUO in both groups, such that reduction in the glomerular filtration rate was due in large part to factors other than RBF. In conclusion, CPUO in the neonatal guinea pig causes marked alterations of glomerular perfusion. Contralateral nephrectomy prevents the rise in RVR due to CPUO, possibly by preserving perfusion to all nephrons and shifting blood flow to more mature deeper cortical glomeruli. (Pediatr Res 18:1266-1271, 1984)
\end{abstract}

\section{Abbreviations}

GFR glomerular filtration rate

CPUO chronic partial ureteral obstruction

Received March 12, 1984; accepted May 22, 1984.

Requests for reprints should be addressed to Robert L. Chevalier, M.D., Department of Pediatrics, Box 386, University of Virginia Medical School, Charlottesville, Virginia, 22908.

This work was supported by National Institutes of Health Grant 5 S07 RR 05431-21, by a Grant-in-Aid from the American Heart Association, and with funds contributed in part by the American Heart Association, Virginia Affiliate. R.L.C is an Established Investigator of the American Heart Association. Portions of this study were presented at the Annual Meeting of the Society for Pediatric Research San Francisco, CA, May 2, 1984.

\author{
RBF renal blood flow \\ BUN, blood urea nitrogen \\ $R V R$ renal vascular resistance \\ RPF, renal plasma flow \\ Hct, hematocrit \\ $\mathbf{A P}$, arterial pressure
}

Obstruction of the urinary tract is an important cause of rena insufficiency in infancy (15). Complete ureteral obstruction in utero results in a nonfunctional kidney, often with severe dysplasia (6). However, congenital partial urinary tract obstruction often leads to progressive renal insufficiency which may in some cases be prevented or reversed by timely surgical correction (19).

Numerous studies have addressed the physiologic mechanisms leading to decreased GFR during and following relief of complete ureteral obstruction, while relatively few investigations have been made of CPUO (20). Furthermore, as renal mass and hemodynamics are changing rapidly in the early postnatal period, CPUO in the neonate may differ from that in the adult. The present study was therefore designed to answer the following questions: 1) What is the effect of CPUO on postnatal renal and somatic growth? 2) How are intrarenal hemodynamics altered by neonatal CPUO? 3) How is the newborn kidney subjected to CPUO affected by reduced renal mass?

The guinea pig was chosen as the species for'study because, as in the human, nephrogenesis is completed at birth and functional renal maturation proceeds during the first 4 postnatal weeks $(2$, 17). Unilateral partial ureteral obstruction was performed within the first 2 days of life and animals were studied at $3 \mathrm{wk}$ of age, during the time of most rapid superficial nephron maturation.

\section{MATERIALS AND METHODS}

Ninety-four Hartley guinea pigs (46 males and 48 females) were anesthetized with halothane within the first $48 \mathrm{~h}$ of life, and a midline abdominal incision was made in each animal. In 32 guinea pigs (Group I) a 2-mm length of sterilized polyethylene tubing (PE50 or PE60, Clay Adams, Parsippany, NJ) was slit longitudinally and placed around the distal third of the left ureter. To serve as controls, the ureter was left untouched in 16 additional animals studied concurrently: In 31 guinea pigs (Group II), polyethylene tubing was placed around the left ureter as above, and the right kidney was excised after ligating the renal pedicle. Fifteen animals undergoing only right nephrectomy served as controls for this group. The incision was sutured in two layers, and animals were returned to their mothers until weaning 
at 14 days. After 14 days, animals were fed standard guinea pig chow. Both males and females were pooled in each group because there is no difference in somatic or renal growth between sexes in the age range studied (1). Only animals with birth weight 75$130 \mathrm{~g}$ were used, and litter size was reduced when necessary to a maximum of four.

Guinea pigs were fasted with free access to water the night before study at 18-30 days of age. Animals were anesthetized with intraperitoneal sodium pentobarbital, $3 \mathrm{mg} / 100 \mathrm{~g}$ body weight and placed on a thermostatically controlled heating table to maintain rectal temperature at $39 \pm 0.5^{\circ} \mathrm{C}$. Tracheostomy was performed and a jugular vein was cannulated for infusion of $0.9 \%$ saline at $0.3 \mathrm{ml} / 100 \mathrm{~g}$ body weight/h. In Group $\mathrm{I},\left[{ }^{3} \mathrm{H}\right]$ inulin (New England Nuclear, Boston, MA) was added to the saline infusate in a concentration of $10 \mu \mathrm{Ci} / \mathrm{ml}$, while $100 \mu \mathrm{Ci}$ / $\mathrm{ml}$ as added for Group II. The contralateral jugular vein was cannulated for infusion of donor guinea pig plasma at a rate of $0.9 \pm 0.1 \mathrm{ml} / \mathrm{h}$, adjusted to maintain constant Hct as described previously (1). A polyethylene catheter was threaded down the right carotid artery such that the catheter tip lay at the aortic root. Mean AP was continuously recorded from this catheter by means of a Statham 23ID pressure transducer coupled to a Hewlett-Packard 7754B recorder.

The abdominal contents were exposed through midline and left subcostal incisions, and the left kidney was covered with saline-soaked cotton (Group I) or placed in a Lucite cup and bathed in saline maintained at $39 \pm 0.5^{\circ} \mathrm{C}$ (Group II). Ureteral diameter was constant proximal to the obstruction, and external diameter was measured halfway between kidney and bladder using calipers under microscopic visualization. A polyethylene catheter was inserted in the abdominal aorta at the aortic bifurcation and connected to a second pressure transducer. A flared polyethylene cannula was sutured into the bladder for collection of urine. To minimize dead space, bladder volume was reduced by pulling the anterior bladder wall through the ligature around the cannula. Because of low urine flow rates in severely obstructed kidneys of Group I, accurate urine flow measurements could not be made. In this Group, therefore, the right ureter was ligated and severed proximal to the ligation before adding inulin to the infusate, and patency of the left ureter was confirmed by meauring radioactivity of urine collected from the bladder catheter. Blood samples $(100 \mu \mathrm{l})$ were drawn from the carotid artery catheter at the beginning of the experiment for determination of Hct and BUN using a colorimetric diacetyl monoxime method. All blood withdrawn was replaced with an equal volume of donor blood. In Group II, two to four timed urine collections, approximately $30 \mathrm{~min}$ each, were made during micropuncture studies reported separately (4). Blood samples were drawn at the beginning and end of each collection period. In both groups, a $100-\mu 1$ blood sample was obtained from the left renal vein simultaneously with an arterial blood sample for determination of inulin extraction $\left(E_{\text {in }}\right)$ as previously described $(3)$. Blood was not obtained from the right renal vein because its access was prevented by the bulky viscera in this species.

An equal mixture of $50-80 \times 10^{3}$ latex microspheres $9 \pm 1$ and $15 \pm 1 \mu \mathrm{m}$ in diameter, labeled with ${ }^{85} \mathrm{Sr}$ or ${ }^{141} \mathrm{Ce}(3 \mathrm{M}$, St. Paul, MN) were suspended in $10 \%$ dextran and Polysorbate 80 by vigorous agitation (Vortex Genie, Scientific Industries, Bohemia, NY) and ultrasonication (Bransonic 12, Branson Co., Shelton, $\mathrm{CN}$ ). While blood was withdrawn from the abdominal aorta at $0.7 \mathrm{ml} / \mathrm{min}$ using a precision withdrawal pump (Harvard Apparatus, Millis, MA), the microsphere mixture was injected into the carotid catheter and flushed with normal saline. In eight additional guinea pigs of Group I (four control and four with CPUO), blood was also withdrawn from the renal vein at 0.5 $\mathrm{ml} / \mathrm{min}$ simultaneously with aortic blood withdrawal, to measure the fraction of $9-$ and $15-\mu \mathrm{m}$ spheres not trapped by the kidney. After documenting that arterial pressure was not altered by microsphere infusion, $1 \mathrm{ml}$ India ink (Higgins No. 4465, Newark, $\mathrm{NJ}$ ) was infused in the carotid catheter followed by infusion of saturated potassium chloride solution to sacrifice the animal.
The kidney(s) were decapsulated, bisected longitudinally, weighed, and fixed in $10 \%$ formalin solution. Radioactivity of the blood sample and kidneys was measured in a gamma scintillation counter (Beckman Instruments, Irvine, CA). One-half of the kidney was digested in $25 \%$ hydrochloric acid and the number of perfused glomeruli was determined as described previously (2). In the remaining half, five cores, $3 \mathrm{~mm}$ in diameter, were punched out perpendicular to the surface of the kidney. Medullary tissue was cut away and the remaining cortex was divided into equal thirds (outer, middle, and inner) and slices from each level were pooled for measurement of radioactivity as previously described (3). Remaining kidney tissue was weighed before and after drying at $40^{\circ} \mathrm{C}$ for 10 days for calculation of dry/wet ratio.

In four guinea pigs of Group I with severe ureteral obstruction, urine was withdrawn from the dilated ureter by sterile needle puncture, plated on sheep blood and McConkey's agar, and incubated at $37^{\circ} \mathrm{C}$ for $48 \mathrm{~h}$.

Calculations. Inulin clearance was calculated from standard formulas and filtration fraction was given by $E_{\text {in. }}$ RBF was calculated from microsphere data using the relationship $\mathrm{RBF}=$ $K \cdot q / b$, where $K=$ radioactivity of whole kidney, $q=$ withdrawal rate of aortic blood reference sample, and $b=$ radioactivity of reference blood sample. $\mathrm{RPF}$ was calculated as $\mathrm{RPF}=\mathrm{RBF}(1$ $-\mathrm{Hct}$ ), and GFR as GFR $=\mathrm{RPF} \cdot E_{\text {in }}$. RVR was calculated as $\mathrm{RVR}=(\mathrm{AP}-\mathrm{RVP}) / \mathrm{RBF}$, where $\mathrm{RVP}=$ renal venous pressure, assigned a value of $3.5 \mathrm{~mm} \mathrm{Hg} \mathrm{(3).}$

Statistical analysis. Data were evaluated by two-way analysis of variance and Duncan's multiple range test using the Statistical Analysis System (S. A. S. Institute, Inc., Cary, NC). The effects of ureteral obstruction and of contralateral nephrectomy were examined separately, and interaction between these variables was also examined. As birth weight was lower in Group II than Group I, and experimental body weight in control animals is dependent on birth weight $(R=0.81, p<0.001)$, analysis of covariance was also carried out for body weight and kidney weight. This was done by using birth weight as a covariate and examining main effects of ureteral obstruction and uninephrectomy and their interaction.

Pearson's correlation was used to evaluate the relationship between body weight and BUN, and linear regression analysis was used to examine the effect of ureteral obstruction on cortical distribution of microspheres in each group. In addition, homogeneity of slopes of the linear relationships was tested between Groups I and II (11).

\section{RESULTS}

At the time of study, the degree of hydroureteronephrosis resulting from ureteral stenosis was variable, and was defined as mild (ureteral diameter under $3 \mathrm{~mm}$ ) or severe (ureteral diameter at least $3 \mathrm{~mm}$ ). As shown in Table 1, birth weight did not differ between obstructed and control animals of either group, but was $8 \%$ lower in Group II than Group I. This difference was taken into account for statistical comparisons of experimental body weight and kidney weight between Groups I and II (Table 1).

Influence of severity of CPUO. As shown in Table 1, CPUO in Group I resulted in decreased body weight, independent of severity of obstruction. In Group II, reduction in somatic growth was more marked in severe than mild CPUO. As shown in Figure 1, somatic growth rate of animals in Group I was not different from controls after the 1st wk of life, although CPUO resulted in smaller body size. In Group II, CPUO eventually resulted in death of the animal, which occurred in the first 30 days with severe obstruction, and was delayed beyond 50 days in the most mild cases. In all animals of Group II, growth rate was normal initially, with growth failure developing acutely before death 1020 days later (Fig. 1).

In Group I, there was no difference between left and right dry kidney weight of sham-operated animals while the affected kidney was significantly smaller than the contralateral one in those with severe but not mild CPUO. For Group I, dry mass of the 


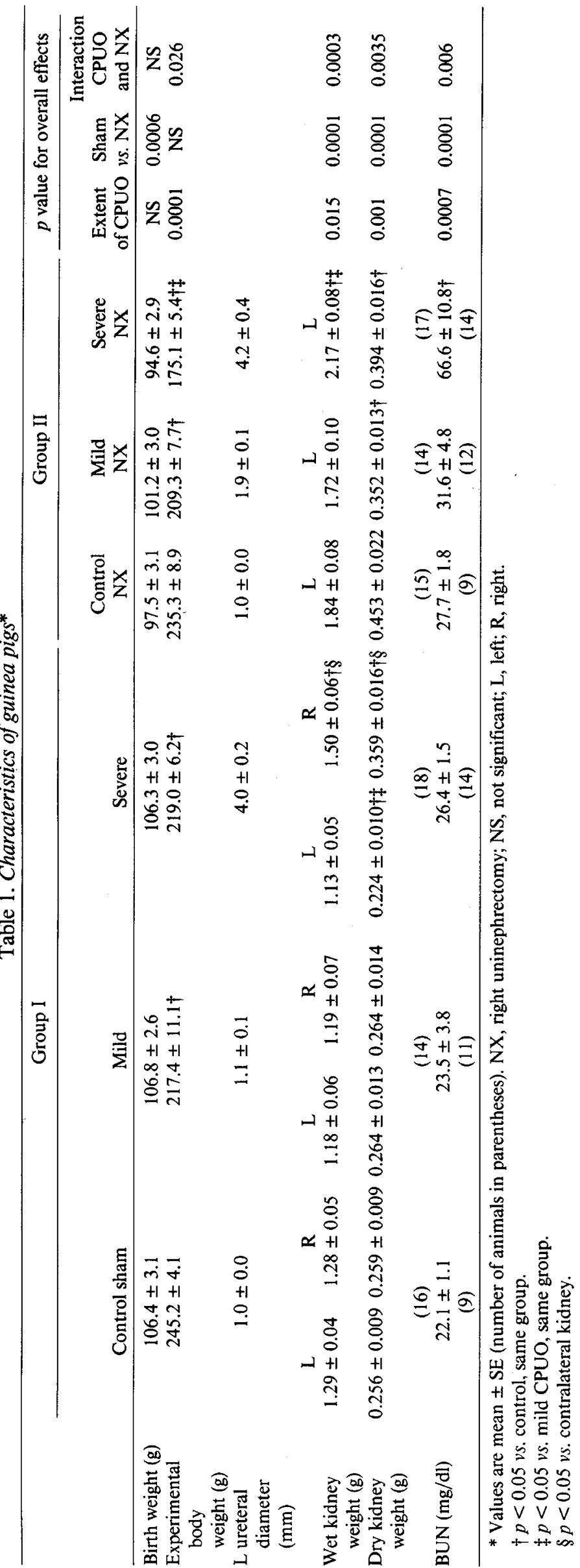

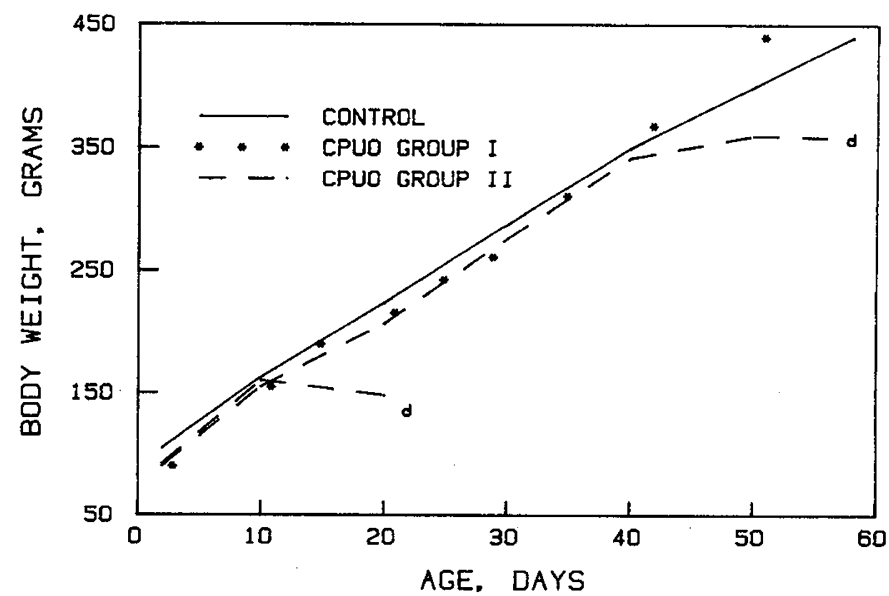

Fig. 1. Somatic growth of representative male animals; $d$ is death of animal from renal failure. Two guinea pigs with CPUO from Group II are depicted: one with mild obstruction dying at 56 days of age and one with severe CPUO dying at 21 days of age.

obstructed kidney of animals with severe CPUO was less than that of control or mild CPUO groups, and the contralateral kidney was significantly larger. As a result of CPUO in Group II, dry kidney weight was less than that of controls, but did not vary with severity of obstruction. There was no effect of unilateral CPUO on BUN of Group I, while in Group II, BUN increased with severity of obstruction.

In all animals of Group I subjected to CPUO, significant inulin activity was measured in urine collected distal to the obstruction of the left ureter, indicating that obstruction was partial rather than complete, although urine flow was too slow for accurate measurement. In Group II, urine flow was $14.9 \pm 4.6 \mu \mathrm{l} / \mathrm{min}$ for controls and did not differ between that of animals with mild $(12.1 \pm 2.8 \mu \mathrm{l} / \mathrm{min})$ or severe CPUO $(16.9 \pm 3.0 \mu \mathrm{l} / \mathrm{min})$. There was no difference between RBF calculated from inulin clearance and that measured by $15-\mu \mathrm{m}$ microspheres in control animals of Group II. However, RBF calculated from inulin clearance was $33 \pm 10 \%$ lower in those with CPUO $(p<0.01)$. Calculation of $\mathrm{RBF}$ using $15-\mu \mathrm{m}$ microspheres was $6 \pm 1 \%$ greater than that using 9- $\mu \mathrm{m}$ spheres $(p<0.001)$. Furthermore, recovery of 15 $\mu \mathrm{m}$ spheres in the renal vein averaged $0.06 \pm 0.03 \%$ of $\mathrm{RBF}$ while that of $9-\mu \mathrm{m}$ spheres was $2.6 \pm 1.1 \%$. For both groups, the proportion of $15-\mu \mathrm{m}$ microspheres trapped in the outer cortex was $6 \pm 1 \%$ higher than that of $9-\mu \mathrm{m}$ spheres $(p<0.001)$.

Because extraction of microspheres by the kidney was complete for $15-\mu \mathrm{m}$ but not 9- $\mu \mathrm{m}$ spheres, the former were used for measurement of total RBF. As shown in Table 2, mean arterial blood pressure did not differ between groups or as a result of CPUO. In Group I, RBF decreased (and RVR increased) with severe but not mild CPUO. The number of perfused glomeruli in the unobstructed right kidney increased in proportion to the severity of left CPUO in this group $(p<0.001)$. In group II, RBF, RVR, and number of perfused glomeruli were unaffected by CPUO. Filtration fraction and GFR fell as a result of severe but not mild CPUO in each group.

As shown in Figure 2, in Group I there was no effect of CPUO on left kidney outer cortical distribution of 9- $\mu \mathrm{m}$ spheres, while a decrease of inner cortical distribution with increasing severity of CPUO was of borderline significance. As shown in Figure 3, increasing severity of CPUO in Group I was associated with an increase in left kidney outer cortical and decrease in inner cortical distribution of $15-\mu \mathrm{m}$ spheres. In the right kidney of Group I, there was an increase in outer cortical distribution of $9-\mu \mathrm{m}$ spheres with increasing left CPUO (Fig. 2). In Group II, increasing severity of CPUO was associated with a significant decrease in outer cortical distribution of $9-\mu \mathrm{m}$ spheres while the change in $15-\mu \mathrm{m}$ spheres was not significant (Figs. 2 and 3). 


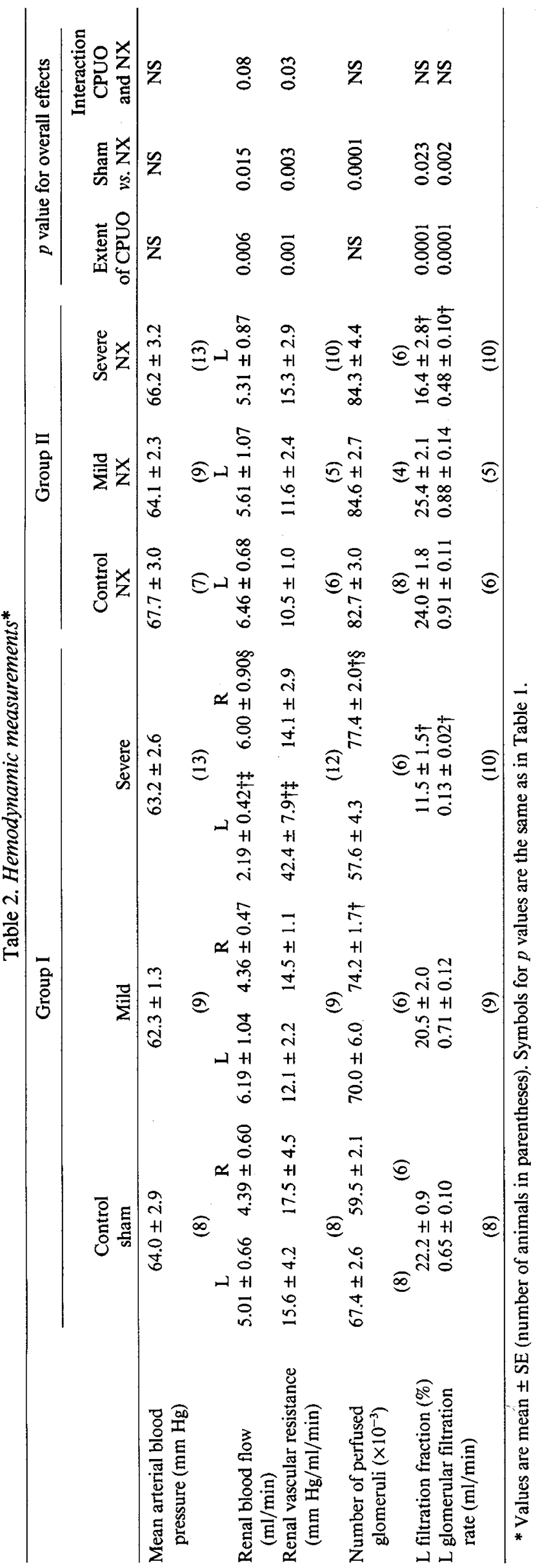

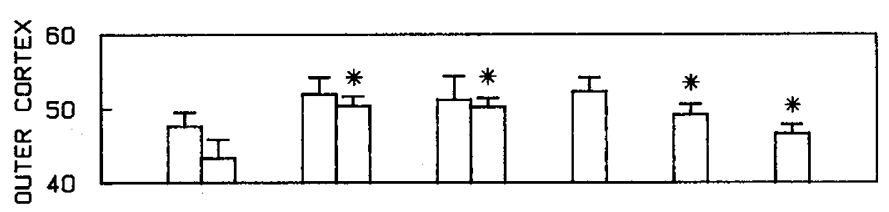
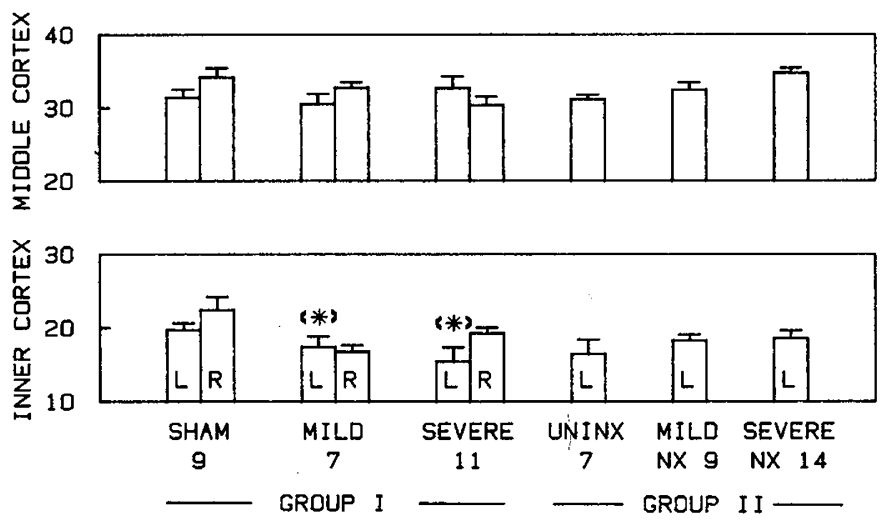

Fig. 2. Cortical distribution of $9-\mu \mathrm{m}$ spheres expressed as percentage of total cortical activity. Number of animals below each category. Uninx, control uninephrectomy; $N X$, contralateral uninephrectomy. For each cortical level and each kidney of Groups I and II, the effect of CPUO was evaluated by linear regression analysis. * indicates a slope different from zero, i.e. an increase or decrease as a function of severity of CPUO $(p<0.05) .<^{*}>$ indicates $p=0.05$.
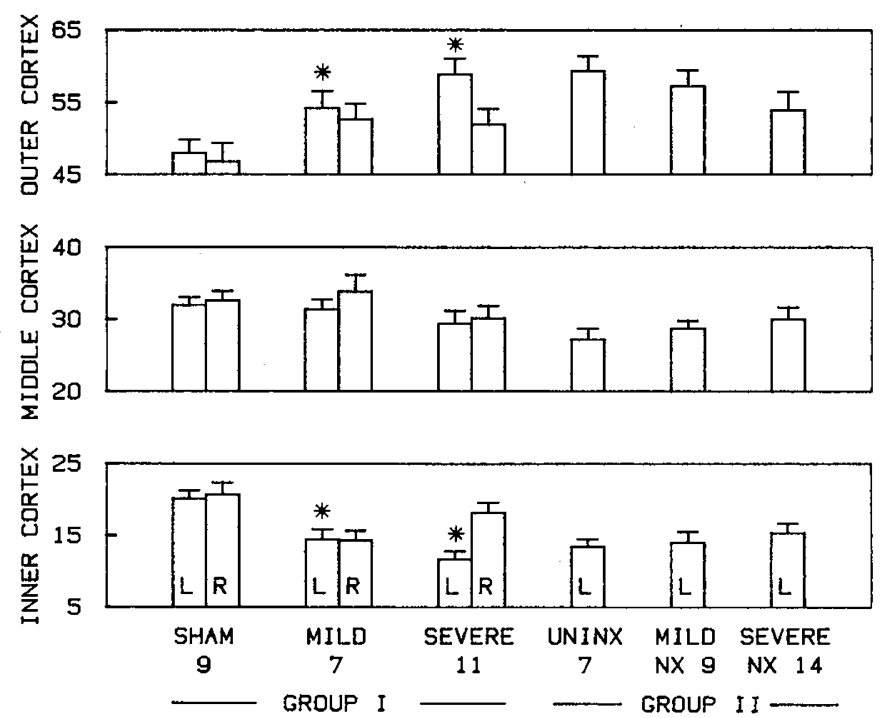

Fig. 3. Cortical distribution of $15-\mu \mathrm{m}$ spheres. Notations are the same as in Figure 2.

Influence of uninephrectomy. Removal of the contralateral kidney resulted in a greater reduction in somatic growth during CPUO (Table 1). Compared to the left kidney of control guinea pigs in Group I, dry weight of the remaining kidney was higher in Group II animals. Dry weight of the right kidney of animals with severe CPUO (Group I) was lower than that of the remaining left kidney of control animals in Group II $(p<0.001)$.

Left kidney RBF was higher and RVR lower in Group II compared to Group I, with the most marked difference present in animals with severe CPUO (Table 2). The number of perfused glomeruli in the single kidney of Group II exceeded that of the left kidney of Group I animals. In addition, overall filtration fraction and GFR were significantly higher in Group II than Group I. Unlike the effect of uninephrectomy to further reduce RVR in animals with severe CPUO, no interaction of these variables was found for filtration fraction or GFR (Table 2). 
Changes in cortical RBF distribution with severity of CPUO were in the opposite direction for Group II compared to the obstructed kidney of group I. The decrease in outer cortical distribution of $9-\mu \mathrm{m}$ spheres with increasing severity of CPUO in Group II differed from the lack of effect on left kidney outer cortical distribution of Group I $(p<0.05)$ (Fig. 2). The increase in left kidney outer cortical distribution of $15-\mu \mathrm{m}$ spheres with increasing severity of CPUO in Group I differed from the lack of effect of CPUO on distribution of spheres in the remaining kidney of Group II $(p<0.001)$ (Fig. 3).

\section{DISCUSSION}

Effects of CPUO on somatic and renal growth. As shown in the present study, neonatal unilateral CPUO results in impairment of somatic growth even when the contralateral kidney is normal. Similar observations were reported by Taki et al. (18), who found that body weight was reduced $4 \mathrm{wk}$ following unilateral CPUO regardless of the age of inducing ureteral stenosis. Furthermore, children with severe unilateral or bilateral vesicoureteral reflux have decreased physical growth which normalizes after surgical correction of reflux (12). As uninephrectomy alone did not result in growth impairment, reduction in renal mass cannot be implicated and there was no evidence of urinary tract infection in any of the guinea pigs of our study. Because animals were not weaned until 14 days of age, caloric intake could not be quantitated in our animals. It is nonetheless possible that ureteropelvic distension due to CPUO in Group I animals resulted in decreased nutrition and lower body weight, but the caloric deficit was not severe enough to compromise growth velocity. When severe CPUO developed in animals with a single kidney, growth was further impaired. Because previous studies in this laboratory have shown no effect of uninephrectomy on somatic growth in guinea pigs of identical birth weight (1) and there was no difference in birth weight as a function of CPUO in either group, it is unlikely that the slightly lower birth weight of Group II had an effect on growth separate from that taken into account by covariate analysis. Cessation of growth prior to death presumably reflects development of "critical" stenosis due to severe dilatation and convolution of the ureter and onset of uremia. As suggested by a study of CPUO in young and adult guinea pigs (18), maturing nephrons appear to be more susceptible to damage due to ureteral obstruction.

Compensatory hypertrophy of the right kidney in animals of Group I with severe CPUO of the left kidney was less than the adaptive growth observed in control uninephrectomized guinea pigs of Group II. This difference probably results from the small but significant functional capacity of the obstructed kidney, and reduced "counterbalance" by the unobstructed organ (8). As dry kidney weight of animals in Group II with severe CPUO was $50 \%$ greater than that of control animals in Group I, compensatory hypertrophy occurred despite the presence of obstruction. In Group II, diminished dry kidney weight compared to controls may be explained by impairment of compensatory hypertrophy by CPUO. Alternatively, the uremic factor(s) responsible for diminished body growth may reduce renal growth as well.

Technical considerations. Using a similar protocol for measurement of RBF in uninephrectomized and sham-operated neonatal guinea pigs (3), determination of RBF by $15-\mu \mathrm{m}$ microspheres correlated well with clearance technque $(r=0.99, p<$ 0.01 ). Thus, although microsphere injection followed urine collection periods, the correlation of RBF determinations suggests that blood flow was relatively constant during the experiment. In addition to providing an accurate measure of RBF in the face of ureteral dilatation, use of radioactive microspheres also permits evaluation of intracortical distribution of blood flow. However, recent studies have shown that compared to $10-\mu \mathrm{m}$ spheres, $15-\mu \mathrm{m}$ spheres in the rat may underestimate inner cortical and medullary blood flow (5). Because the magnitude of skimming artifact is dependent on sphere size, use of smaller spheres (7-10 $\mu \mathrm{m})$ has been advocated for measurement of intrarenal blood flow distribution $(14,16)$. In the present study, a consistent difference in outer cortical perfusion measured with 9- and 15$\mu \mathrm{m}$ spheres injected simultaneously indicates the presence of such artifact. As vasodilatation reduces skimming of 15- more than that of 9- $\mu \mathrm{m}$ spheres (16), distribution of both sizes was examined in the present study to corroborate the direction of change in cortical RBF distribution with varying CPUO.

Renal hemodynamics. While severe CPUO resulted in a marked increase of RVR in Group I, contralateral nephrectomy prevented this rise. In a study of the adult rat subjected to unilateral CPUO at birth, RBF was reduced by only $22 \%$ compared to the contralateral kidney (10). The effect of contralateral uninephrectomy on RBF in unilateral CPUO has not been previously reported, although $\mathrm{RBF}$ is reduced to a similar extent $24 \mathrm{~h}$ following unilateral or bilateral complete ureteral obstruction (13). Silicone rubber injection studies in the adult rat following release of 24-h complete ureteral obstruction suggested patchy vasoconstriction at the level of interlobular arteries (7). A similar phenomenon may explain the $20-30 \%$ reduction in number of perfused glomeruli observed in animals of Group I compared to Group II. We have previously shown that approximately $20 \%$ of glomeruli are underperfused in the normal guinea pig until after $3-4 \mathrm{wk}$ of age, and that complete perfusion occurs by $3 \mathrm{wk}$ of age when uninephrectomy is performed at birth (2). Increase in number of perfused glomeruli of the right kidney in Group I with increasing severity of obstruction is presumably due to the same phenomenon of compensatory vasodilation following vasoconstriction of the obstructed kidney. This suggests that control of renal counterbalance (8) takes place at the level of individual glomerular perfusion. Current evidence favors a role for endogenous vasoconstrictors and vasodilators in determining $R B F$ during ureteral obstruction $(9,21)$. In CPUO, the net balance between vasoactive hormones appears to determine resulting vascular resistance (9) such that vasoconstrictors may predominate in Group I while vasodilators counter their effect in Group II. While arteriolar tone most likely determines RBF during CPUO, vascular diameters were not measured in the present study, and it is possible that intrarenal venous pressure also modulates total RBF.

If distribution of microspheres is taken as a qualitative index of RBF distribution, CPUO in Group I resulted in greater vasoconstriction of inner than outer cortex. As infusion of vasoconstrictors results in a similar pattern of redistribution in the dog kidney (5), it is likely that locally produced vasoconstrictors are responsible for the shift during severe CPUO in Group I. The finding of redistribution of $9-\mu \mathrm{m}$ spheres to deeper cortical regions with increasing severity of CPUO in Group II animals cannot be ascribed to changes in total RVR .However, as proposed above, endogenous vasodilators may exert a greater influence on renal hemodynamics following contralateral nephrectomy, and pharmacologic renal vasodilation causes a similar shift to deeper cortical levels (5).

An overall increase in filtration fraction following uninephrectomy and decrease in this parameter with severe CPUO indicates that GFR is largely dependent on factors other than RBF. Approximately half of the reduction of GFR in animals with severe CPUO in Group I may be explained by reduced RBF while decreased ultrafiltration coefficient and effective filtration pressure are primarily responsible for lowering of GFR in Group II (4).

In summary, unilateral CPUO in the newborn guinea pig results in hemodynamic alterations which are significantly influenced by the presence or absence of the contralateral kidney. Renal blood flow was found to decrease with increasing severity of ipsilateral ureteral obstruction, while the number of perfused glomeruli increased in the contralateral kidney which exhibited compensatory hypertrophy. Despite severe CPUO, uninephrectomized animals developed compensatory renal hypertrophy and RBF did not decrease significantly. Redistribution of RBF to 
outer cortical regions with ipsilateral CPUO in Group I suggests the action of circulating vasoconstrictors. In contrast, distribution of RBF to inner cortical regions following uninephrectomy and severe CPUO in Group II may be due to the opposing actions of vasodilators. A better understanding of the factors underlying changes in glomerular perfusion patterns during CPUO in early development may lead to better preservation of renal function in infants with congenital obstructive nephropathy.

Acknowledgments. Ms. Jean De Piro provided secretarial assistance.

\section{REFERENCES}

1. Chevalier RL 1982 Functional adaptation to reduced renal mass in early development. Am J Physiol 242:F190

2. Chevalier RL 1982 Glomerular number and perfusion during normal and compensatory renal growth in the guinea pig. Pediatr Res 16:436

3. Chevalier RL 1983 Hemodynamic adaptation to reduced renal mass in early postnatal development. Pediatr Res 17:620

4. Chevalier RL Chronic partial ureteral obstruction in the neonatal guinea pig. II. Pressure gradients affecting glomerular filtration rate. Pediatr Res 18:1271

5. Clausen G, Tyssebotn I, Kirkebo A, Ofjord ES, Aukland K 1981 Distribution of blood flow in the dog kidney. III. Local uptake of $10 \mu \mathrm{m}$ and $15 \mu \mathrm{m}$ microspheres during renal vasodilation and constriction. Acta Physiol Scand 113:471

6. Griscom NT, Vawter GF, Fellers FX 1975 Pelvoinfundibular atresia: the usual form of multicystic kidney: 44 unilateral and two bilateral cases. Semin Roentgenol 10:125

7. Harris RH, Yarger WE 1974 Renal function after release of unilateral ureteral obstruction in rats. Am J Physiol 227:806

8. Hinman F 1923 Renal counterbalance: an experimental and clinical study with reference to the significance of disuse atrophy. J Urol 9:289

9. Ichikawa I, Brenner BM 1979 Local intrarenal vasoconstrictor-vasodilator interactions in mild partial ureteral obstruction. Am J Physiol 236:F131

10. Josephson S, Wolgast M, Ojteg G 1982 Experimental obstructive hydronephrosis in newborn rats. II. Long-term effects on renal blood flow distribution. Scand J Urol Nephrol 16:179

11. Kleinbaum DG, Kupper LL 1978 Applied Regression Analysis and Other Multivariable Methods. Duxbury Press, North Scituate, MA, pp 97-102

12. Merrell RW, Mowad JJ 1979 Increased physical growth after successful antireflux operation. J Urol 122:523

13: Moody TE, Vaughan ED Jr, Gillenwater JY 1977 Comparison of the renal hemodynamic response to unilateral and bilateral ureteral occlusion. Invest Urol 14:455

14. Morkrid L, Ofstad J, Willassen Y 1978 Diameter of afferent arterioles during autoregulation estimated from microsphere data in the dog kidney. Circ Res 42:181

15. Reimold EW, Don TD, Worthen HG 1977 Renal failure during the first year of life. Pediatrics 59 (suppl):987

16. Sabto J, Bankir L, Grunfeld JP 1978 The measurement of glomerular blood flow in the rat kidney: influence of microsphere size. Clin Exp Pharmacol Physiol 5:559

17. Spitzer A, Brandis M 1974 Functional and morphologic maturation of the superficial nephrons: relationship to total kidney function. J Clin Invest 53:279

18. Taki M, Goldsmith DI, Spitzer A 1983 Impact of age on effects of ureteral obstruction on renal function. Kidney Int 24:602

19. Warshaw BL, Hymes LC, Woodard JR 1982 Long-term outcome of patients with obstructive uropathy. Pediatr Clin N Am 29:815

20. Wright FS 1982 Effects of urinary tract obstruction on glomerular filtration rate and renal blood flow. Semin Nephrol 2:5

21. Yarger WE, Schocken DD, Harris RH 1980 Obstructive nephropathy in the rat: possible roles for the renin-angiotensin system, prostaglandins, and thromboxanes in postobstructive renal function. J Clin Invest 65:400

\title{
Chronic Partial Ureteral Obstruction in the Neonatal Guinea Pig. II. Pressure Gradients Affecting Glomerular Filtration Rate
}

\author{
ROBERT L. CHEVALIER \\ With the technical assistance of Anthony V. Broccoli \\ Department of Pediatrics, University of Virginia School of Medicine, Charlottesville, Virginia 22908
}

\begin{abstract}
Neonatal guinea pigs with chronic partial ureteral obstruction (CPUO) and contralateral nephrectomy develop hydroureteronephrosis and reduced glomerular filtration rate (GFR) without significant reduction of renal blood flow. To investigate the role of pressure gradients in determination of GFR, micropuncture studies were performed in animals $23 \pm 3$ days of age subjected to
\end{abstract}

Received March 12, 1984; accepted May 22, 1984.

Requests for reprints should be addressed to Robert Chevalier, M.D., Department of Pediatrics, Box 386, University of Virginia Medical School, Charlottesville, Virginia 22908.

This work was supported by National Institutes of Health Grant 5 S07 RR 05431-21, by a Grant-in-Aid from the American Heart Association, and with funds contributed in part by the American Heart Association, Virginia Affiliate. R.L.C. is an Established Investigator of the American Heart Association. This study was presented in part at the Second International Workshop on Developmental Renal Physiology, Marburg, West Germany, August 27, 1983, and at the 6th Internationa Pediatric Nephrology Symposium, Hanover, West Germany, September 1, 1983. left ureteral constriction and right nephrectomy within the first 2 days of life and compared to uninephrectomized controls. Resulting ureteral dilatation was variable, with kidney weight and ureteral diameter being proportional to the rise in ureteral pressure $\left(P_{U}\right)$. In individual animals with severe CPUO (ureteral diameter $\geq 3 \mathrm{~mm}$ ), distal tubular transit time was either normal (31-90 s) or prolonged (>120 s). Superficial single nephron GFR (SNGFR) was inversely correlated with $\boldsymbol{P}_{U}$.

Glomerular capillary pressure and afferent arteriolar colloid oncotic pressure were not affected by CPUO while peritubular capillary, proximal and distal intratubular hydrostatic pressure increased as a function of $P_{U}$. As a result, afferent effective filtration pressure $\left(\boldsymbol{E F P} \boldsymbol{P}_{A}\right)$ was reduced in severe $(10.0 \pm 1.1 \mathrm{~mm} \mathrm{Hg})$ compared to mild CPUO (13.4 $\pm 0.5 \mathrm{~mm} \mathrm{Hg}$ ), but was not different from controls (11.3 $\pm 0.9 \mathrm{~mm} \mathrm{Hg}$ ). For both control and CPUO groups, 